\title{
Sheath size and Child-Langmuir law in one dimensional bounded plasma system in the presence of an oblique magnetic field: PIC results
}

\author{
J. Moritz,, a) S. Heuraux, E. Gravier, M. Lesur, F. Brochard, L. De Poucques, E. \\ Faudot, and N. Lemoine \\ Institut Jean Lamour, UMR 7198 CNRS - Université de Lorraine, Campus Artem, \\ 2 allée André Guinier, 54011 Nancy, France.
}

(Dated: 21 June 2021)

The sheath properties are studied by using $1 \mathrm{~d} 3 \mathrm{~V}$ particle-in-cell simulations in a plasma bounded by two conductive electrodes between which is applied a constant voltage, $V_{w}$. A magnetic field tilted by $\theta$ with respect to the wall is considered in the simulations. Elastic collisions with neutrals are modeled by an operator that randomly reorients ions and electrons in the velocity space. The ratio between the ion mean-free-path and the Larmor radius is chosen such that $\frac{\lambda_{c i}}{R}>1, \simeq 0.5$ or $<1$, whereas the same ratio for electrons is always $\gg 1$. For low ion collisionality $\left(\frac{\lambda_{c i}}{R}>1\right)$ and incidences such that $\sin \theta>\frac{R}{\lambda_{c i}}$, the sheath size is shown to scale with $\sin \theta$ and depends on $V_{w}$ according to the Child-Langmuir law, with a $3 / 4$ exponent. For larger collisionalities, the $\sin \theta$ dependence of the sheath size disappears because ions are demagnetized by collisions. Then, for incidences $\theta>5^{\circ}$, the sheath size varies with a $3 / 5$ exponent of the wall potential, as expected in moderate collisional sheaths. More interestingly, for grazing incidences and $\frac{\lambda_{c i}}{R} \simeq 0.5$, an inverse sheath, ie. an electro-negative space charge, arises at the wall vicinity in order to screen the positive wall potential (instead of the negative one). Its size, comparable to a classical Debye sheath, is shown to vary with a $2 / 3$ exponent of the wall potential. Finally, our simulation results show that the Child-Langmuir law is a good way to evaluate the sheath size for a large range of collisionality at any magnetic field incidence as long as the exponent is chosen accordingly.

a)jerome.moritz@univ-lorraine.fr 


\section{INTRODUCTION}

The circulating current in a plane-parallel vacuum diode is strongly limited by space charge effects, even if the reservoir of charges - positive or negative - on the plates can be considered as infinite. The first model explaining this electrostatic effect is due to $\mathrm{Child}^{1}$ in 1911, where he calculated the current carried by positive ions, for a given distance from the anode, and for a given potential difference between the plates, $V$. The same approach was used by Langmuir ${ }^{2}$ two years later, for electrons as the charge carriers, in order to explain the saturation of thermionic currents with the temperature of an emitted filament. Simply stated, for particles emitted without initial velocity from a plate, as soon as the electric field is canceled at the plate due to charge effects, the current is limited. This basic assumption allows the derivation of the well-known Child-Langmuir expression of the current:

$$
J=\frac{4 \epsilon_{0}}{9} \sqrt{\frac{2 e}{m_{p}}} \frac{V^{3 / 2}}{L^{2}},
$$

where $J$ is the maximum charge current, $e$ the electron charge, $m_{p}$ the mass of the particle and $L$ the distance between electrodes.

Eq.(1) applies in vacuum, but can also be used in plasma to characterize the current crossing a sheath developed at the vicinity of a biased wall, or to determine its spatial extension with respect to the wall potential, which is important for instance in Langmuir probe measurements and their interpretation. The sheath is in fact a thin layer of positive charge built up over several Debye lengths in front of any surface plunged into a plasma, whose role is to accelerate ions and push back electrons, in order to balance the particles fluxes. Assuming a zero potential as well as a zero electric field at the sheath entrance, and ions entering the sheath with a negligible velocity (vs. the one acquired inside the sheath itself), we can derive an expression similar to Eq. (1), where the distance between electrodes is now the sheath size, $s$, as:

$$
\phi_{w}^{3 / 4}=\frac{3}{2}\left(\frac{J_{i}}{\epsilon_{0}}\right)^{1 / 2}\left(\frac{2 e}{M}\right)^{-1 / 4} s,
$$

with $\phi_{w}$ the amplitude of the wall potential, $M$ the ion mass and $J_{i}$ the ion current entering the sheath. In order to neglect the electron contribution to the space charge and solve easily the Poisson equation, one had to assume that $k_{b} T_{e} \ll\left|e \phi_{w}\right|$, where $T_{e}$ is the 
electron temperature, so that the electron density $n_{e} \simeq 0$ within a large portion of the sheath.

This last expression, also known as the Child-Langmuir law, shows that the sheath varies with a $3 / 4$ power dependence on the surface potential, and depends on the ion current coming from the plasma and crossing the sheath. In the latter description, the sheath was assumed collisionless, which is not always the case in high pressure plasma, especially because of charge exchange collisions ${ }^{3}$. Different regimes of the ion collisionality can be considered depending on $\frac{v_{i x}}{v_{t i}}$, the ion drift (perpendicular to the wall) to the ion thermal velocity ratio ${ }^{4}$. If between two subsequent collisions the energy gained by ions in the electric field $E$ is small with respect to the thermal energy, then $v_{i x} \ll v_{t i}$, and the collision rate is given by $\nu_{i}=\frac{v_{t i}}{\lambda_{c i}}$, with $\lambda_{c i}$ the ion-neutral collision mean free path. However, if ions are strongly accelerated between two collisions, we have $v_{i x} \gg v_{t i}$ and $\nu_{i} \simeq \frac{v_{i x}}{\lambda_{c i}}$. In the first case corresponding to high pressure plasmas or high ion collisionality, the ion mobility in the electric field is $\mu_{i}=\frac{e}{\nu_{i} M}$ and is independent of the drift velocity. Assuming that within the sheath, the ion current is given by $J_{i}=e n_{i} \mu_{i} E$, it is straightforward to integrate the Poisson equation using the boundary condition $E(s)=-\phi^{\prime}(s)=0$ in order to obtain a $2 / 3$ power dependence $^{5}$ on the surface potential for the sheath size $s$. In the moderate collisionality case, the ion current crossing the sheath is given by $J_{i} \simeq e n_{i} \sqrt{\frac{e \lambda_{c i} E}{M}}$, and the variation of $s$ with $\phi_{w}$ is rather a $4 / 5$ power dependence ${ }^{6}$.

A similar remark applies to the upstream ion current $J_{i}$ entering the sheath, which also depends on the collisionality. The stability of the sheath requires that, for a plasma where $T_{i}$ and $T_{e}$ are the ion and electron temperatures respectively, the ion velocity perpendicular to the wall, $v_{i x}$, has to be super-sonic $v_{i x} \geq c_{s}$, with $c_{s}=\sqrt{\frac{k_{b}\left(T_{i}+T_{e}\right)}{M}}$, which is known as the Bohm criterion ${ }^{7}$. In the pre-sheath, a quasi-neutral region of the plasma, ions are accelerated up to the sonic point, where the plasma approximation $n_{i} \simeq n_{e}$ breaks down ${ }^{8,9}$. It is then straightforward to define the sheath entrance as the point where ions reach $c_{s}$; then $J_{i}$ is proportional to $c_{s}$ with $J_{i}=\alpha e n_{0} c_{s}$ and $n_{0}$ is the plasma density. The value of $\alpha$ depends on the collisionality, as does the surface potential exponent, larger is the collisionality, smaller is $\alpha$. In a low pressure plasma, the simplest fluid model taking into account ionization processes in the pre-sheath leads to $\alpha \simeq 0.6$, while the kinetic treatment of the new born ions by Tonk and Langmuir ${ }^{10}$, further reexamined by Harrison and Thompson and other authors ${ }^{11,12}$, gives $\alpha \simeq 0.426$. On the opposite pressure limit, $\alpha$ is expected to be smaller ${ }^{13,14}$ and depends on 
the ratio between the ion-neutral collision mean-free-path $\lambda_{c i}$ and the plasma length $L$.

In addition, $\alpha$ also depends directly on the presence of an oblique magnetic field, tilted by $\theta$ with respect to the walls, in the low collisionality regime. In this oblique $B$ case, another plasma layer forms between the Debye sheath and the collisional pre-sheath. This additional layer, usually called Chodura sheath ${ }^{15}$ or magnetic pre-sheath, scales with the ion Larmor radius $R$, and its role is to reorient the ion flux from a direction parallel to the magnetic line to a direction perpendicular to the wall at $v_{i x}=c_{s}$. When collisions can be neglected, it was demonstrated that the ion velocity along the magnetic field reaches $c_{s}$ at the Chodura sheath entrance (ie. $c_{s} \sin \theta$ in the $x$ direction), which is equivalent to a Bohm criterion along $B$, although the plasma approximation $n_{i} \simeq n_{e}$ still stands in this specific region ${ }^{16-18}$. Consequently, the drop of density in the Chodura sheath is expected to scale with $\sin \theta$. The ion current $J_{i}$ crossing the sheath decreases with decreasing incidence of $B$ with respect to the wall ${ }^{19}$.

However, both collisions with neutrals and very grazing incidences can impact the angular trend of $J_{i}$ described above and yet the existence of the Debye sheath. Concerning the ionneutral collisions, they induce a motion perpendicular to the magnetic line, which destroys the anisotropy of the velocity field when $\lambda_{c i}<R$ and so the Chodura sheath. But even for smaller collisionality, ie. $\lambda_{c i}>R$, since the Chodura sheath extent in front of the wall is of about one ion Larmor radius, when the magnetic field incidence is such that $\theta<\arcsin \left(R / \lambda_{c i}\right)$, collisions occur within the magnetic pre-sheath. The latter then merges with the collisional one ${ }^{18,20}$. Therefore the ion current $J_{i}$ crossing the sheath is not expected to scale with $\sin \theta$ for such grazing incidences and must be described by a collisional model. Finally the sheath itself might not form when the incidence of the magnetic field is so small that ions are collected mainly perpendicularly to the $B$ direction in a time scaling with $\omega_{c i}$, while electrons, much more constrained to follow the field line, need as much time (or more) to connect the wall. A straightforward calculation based on geometrical considerations gives an angle of the order of $\theta_{l} \simeq \sqrt{\frac{m}{M}}$, where $m$ is the electron mass, in the collisionless case and in floating wall conditions. Note that the same phenomenon exists in the presence of collisions with neutrals and that the critical incidence at which it appears can be larger than $\theta_{l}{ }^{21}$. In the extreme case of $\theta=0$, and in the absence of collisions, the particle fluxes at the walls cancel, which can lead to the presence of an electro-negative charge at the wall vicinity due to the difference between the ion and electron Larmor radii and whose spatial extension 
scales between $\lambda_{d}$ and $R^{22-24}$. Turbulence can restore the particle current perpendicularly to the field line though, but in 2D systems only, due to a $E \times B$ velocity shear in the sheath region $^{25}$.

More generally, the knowledge of the sheaths physics has increased in recent decades thanks to progresses in computational techniques, including particle-in-cell (PIC) or Vlasov methods, coupled sometimes to fluid models. These previous numerical works have focused on many fundamental aspects of plasma sheaths, from the investigation of the plasma wall transition in the presence of a magnetic field and collisions ${ }^{18,26-28}$, to the calculation of angular and energy distributions of ions impinging on the wall, which is a key parameter of wall erosion and sputtering ${ }^{29,30}$. The effect of secondary electrons emitted from the wall ${ }^{31}$ or of multi ion components onto the sheath potential and structure by PIC simulations ${ }^{32}$ was also addressed as well as the sheath structure in presence of a RF potential applied to the collecting surface ${ }^{33}$. These previous studies emphasized the sheath structure in different conditions of wall emission, angle of incidence of the magnetic field or collision rate with neutrals.

In this paper, we investigate by means of PIC simulations the Debye sheath properties, and more particularly the sheath extent, in the presence of a magnetic field tilted by various angles with respect to the wall, and for different charged particles vs. neutrals collision rates. Our simulations are run with a variable voltage $V_{w}$ applied between the two conductive plates delimiting the simulated plasma. Our main goal is to characterize the sheath size variation vs. $V_{w}$ in order to test the validity of the Child-Langmuir law and determine its most appropriate exponent according to the main models, for a large range of both magnetic field incidence and collisionality. The use of a PIC code and the calculation of the exact motion of electrons, which are not considered at the thermal equilibrium, allows the investigation of very grazing incidences of the magnetic field and of the conditions of existences of classical Debye sheath, which has not been much addressed within the literature

In a first part of the paper, we describe the studied system with a general overview of the PIC code we developed and explain how the different parameters such as the sheath size or the potential drops are extracted from the simulations. In a second part, we focus on the results of the simulations for three values of the mean-free-path to Larmor radius ratios. We show that, in the low collisional case $\left(\lambda_{c i} / R>1\right)$, the sheath size scales with $\sin \theta$ and follows a $3 / 4$ exponent variation of $V_{w}$ as expected, but only for incidences $\theta>\arcsin \left(R / \lambda_{c i}\right)$. 
Then, for larger collisionalities $\left(\lambda_{c i} / R<1\right)$, we show that the $\sin \theta$ dependence disappears and that the exponent of the Child-Langmuir law is closer to 3/5. More interestingly, when large collisionality and grazing incidence are combined, an electro-negative sheath can build up at the wall vicinity (for a strong positive potential of the wall). Using a fluid model, we finally show that the ion sound velocity remains a good criterion for determining the inverse sheath entrance location and that its spatial extension presents a power-law dependence of the applied voltage as well.

\section{PIC SIMULATIONS}

We consider in this study a uni-dimensional plasma bounded by conductive electrodes, between which a constant voltage $V_{w}$ is applied, as depicted in Fig. 1. During the PIC simulations, the right electrode, at $x=L / 2$, is grounded, while the left one at $x=-L / 2$ is set to a positive or a negative potential denoted by $\phi_{w}$ in the rest of this paper. If $\phi_{w}<0$, corresponding to the situation shown in the figure, the ion flow at the left wall, $\Gamma_{i}^{l}$, is expected to be larger than the electron one, $\Gamma_{e}^{l}$. The opposite trend occurs at the right electrode with $\Gamma_{e}^{r}>\Gamma_{i}^{r}$, and the conservation of the total outflow implies that $\Gamma_{e}^{r}+\Gamma_{i}^{r}=\Gamma_{e}^{l}+\Gamma_{i}^{l}$.

While in floating wall conditions the sheath size at the vicinity of the left or the right surface are identical ${ }^{34}$, they depend now on both the sign and amplitude of $\phi_{w}$. In a case similar to that described in Fig. 1, but without magnetic field, the sheath extension at the left electrode $s_{l}$ is expected to be larger than its right counterpart $s_{r}$. Most of the ions are indeed strongly attracted to the left wall, while electrons are repelled inside the plasma, creating a circulating current through the system. The potential drop in the left sheath is approximately $\left|\phi_{w}\right|$ as long as $e\left|\phi_{w}\right|>>k_{b} T_{e}$. Note that in order to determine the sheath extension, we simply search for the position $x_{s}$ where the averaged ion velocity reaches the sonic speed $c_{s}$. Then all sheath parameters, such as its size, the potential or the particle densities at $x_{s}$ can easily be extracted. If for any reason (collisionality, grazing incidence of the magnetic field...), the ion velocity is not supersonic through the simulation domain $x<0$ (resp. $x>0$ ), we assume the absence of a Debye sheath and $s_{l}=0$ (resp. $\left.s_{r}\right)$, even if a small electro-positive charge can be seen at the wall vicinity. The length of the studied plasma is $600 \lambda_{d}$, ion and electron temperatures are set to $k_{b} T_{i}=k_{b} T_{e}=2 \mathrm{eV}$ at the beginning of the simulations. To be consistent with our previous published works and to 


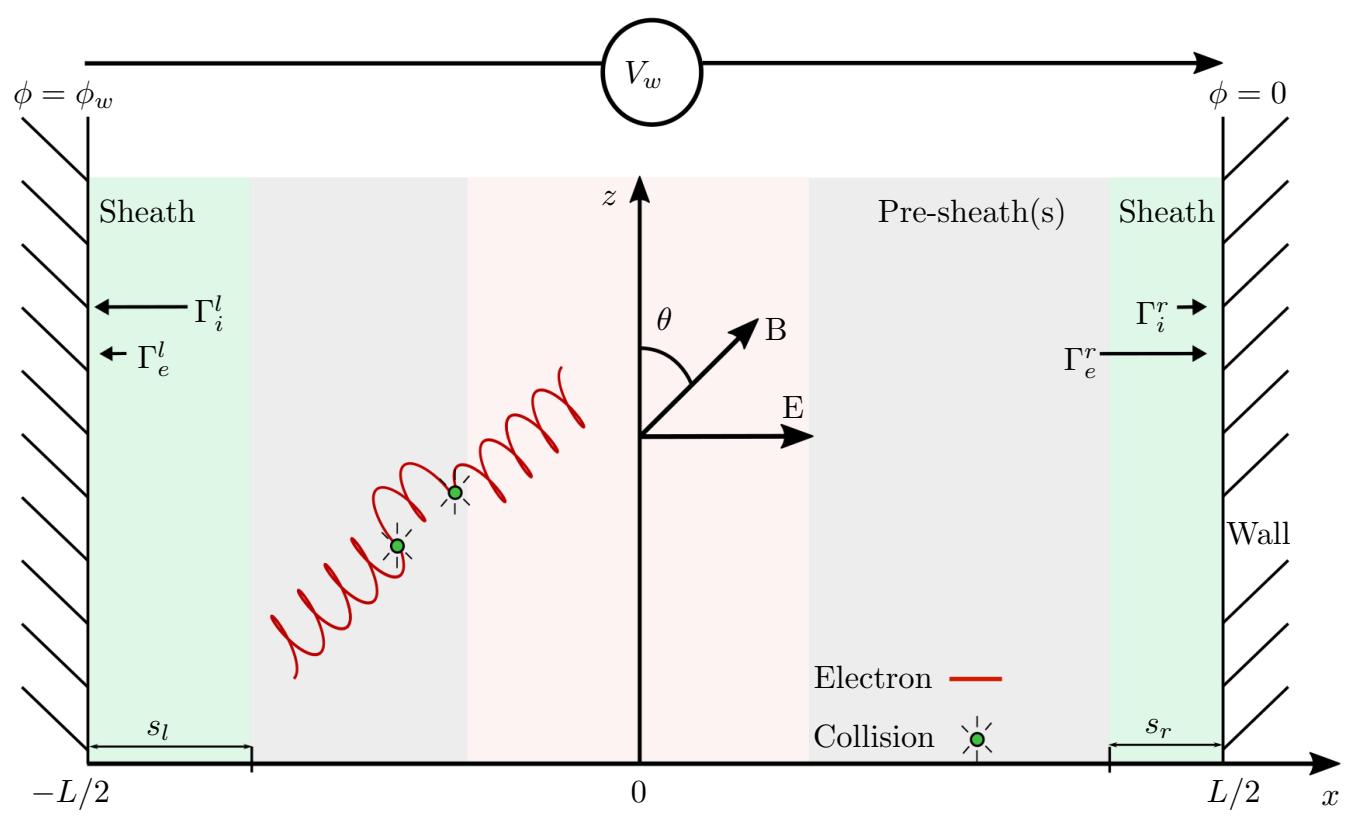

FIG. 1. Sketch of the studied plasma embedded between the two conducting electrodes. The presheath(s) (in gray color), between the bulk plasma at the center and the sheaths entrances identified by $s_{l}$ and $s_{r}$, can be composed successively of a collisional region and a Chodura sheath, or can be only collisional; in both cases, it is a quasi-neutral region of the plasma. In the configuration described in the figure, $\phi_{w}$ is negative.

limit computation time, we employ a reduced ion-to-electron mass ratio of 500 . Indeed in the presence of a tilted magnetic field, the calculations are run for dozens of ion gyro-periods in order to reach the steady state, with a constraint on the time step which has to be small enough for describing the electron gyration around the magnetic lines. A uniform magnetic field tilted by an angle $\theta$ with respect to the $z$ direction is considered in all simulations, giving typical cyclotron-to-plasma frequency ratios of $\omega_{c e} / \omega_{p e}=1.56$ for electrons and of $\omega_{c i} / \omega_{p i}=0.0697$ for ions.

Elastic collisions with neutrals are taken into account during the PIC simulations by using a hard sphere description (see reference ${ }^{35}$ for further details concerning the velocity determination after the elastic collision and the Monte Carlo procedure used). In such a simple model, a constant collision cross-section leads to velocity independent mean-free-path for ions and electrons. Then for each simulation, we set the mean-free-path of the particles as we did in our previous studies, in order to investigate the effect of collisions onto the magnetic pre-sheath ${ }^{21}$. Here we focus on 3 different regimes of collisionality for ions, with $\lambda_{c i} / R=5.5$, 
0.55 and 0.11 ie. low, medium and high collisionality respectively. The corresponding ratios for electrons are $\lambda_{c e} / r=500,50$ and 10. For these specific collisionalities, electrons are always attached to their magnetic field line for several tens of gyroperiods before undergoing a collision, unlike their positive counterpart for the last two ratios. This can lead to interesting situations, particularly for grazing incidences of the magnetic field, where ions can migrate towards the walls as fast as electrons, because of their perpendicular (to the magnetic line) motion induced by collisions as explained previously. During the simulations, a source term is used to maintain a constant number of ions in the studied plasma: when a single ion is absorbed by one of the bounding surfaces, a couple ion + electron is injected randomly within the simulation box according to a uniform law and their velocity chosen in a Maxwellian distribution with the initial temperatures. The number of electrons is simply regulated by the presence of the sheaths.

Concerning the temperature of the particles, it is well known that an artificial cooling arises in PIC codes because energetic particles with larger gyro-radius are absorbed at the wall faster, causing accumulation of low energy ones in the system. It can be avoided by heating up the plasma with artificial methods such that reinitializing the velocity in the nominal distribution within a source region ${ }^{20,28}$, or by changing the injected velocity distribution function ${ }^{36}$. In our study, we did not use such heating procedures so that the actual temperatures can be slightly different than the nominal ones at the end of the simulations for both ions and electrons. In order to determine the sheath entrance location with the sonic speed or to normalize the velocity profiles with respect to $c_{s}$, we extract the local temperatures from the velocity distribution functions for each run independently at the center of the plasma and at the end of the simulations.

The left wall potential $\phi_{w}$ is varied from $+80 \mathrm{~V}$ to $-80 \mathrm{~V}$ for all investigated cases of incidence and collisionality, which gives a ratio $e\left|\phi_{w}\right| / k_{b} T_{e}$ of the order of 40 for the maximum voltage. In the following, we will focus only on the characteristics of the left wall located at $x=-L / 2$ and the sheath size $s_{l}$ will be then simply denoted by $s$ in the rest of the paper. In order to determine the exponent of the Child-Langmuir law for the $s=f\left(\phi_{w}\right)$ characteristics, we try to fit the three exponents of the main available models (ie. $3 / 4,4 / 5$ and $2 / 3$ ) according to the expected collisionality and identify the best one for a series of simulations. 


\section{REGIME OF LOW COLLISIONALITY}

When a large enough negative potential is applied to the left wall $\phi_{w}<-\frac{k_{b} T_{e}}{e}$, for large incidence and low collisionality, the potential drop at the wall vicinity is expected to be of the order of $\phi_{w}$ because of the effective screening of the sheath: electrons are strongly pushed back into the plasma, leading to an electron depletion stronger than in floating wall conditions and $s>s_{0}$, with $s_{0}$ the sheath size for $\phi_{w}=0$. Fig. 2a shows such a situation with $\phi_{w}=-40 \mathrm{~V}$ for $\lambda_{c i} / R=5.5$ and decreasing values of $\theta$. The vast majority of the potential drop extends over several tens of $\lambda_{d}$ in front of the surface and the potential profile exhibits a progressive change as $\theta$ decreases, bending towards the plasma center, because of an increase in the sheath size. This tendency can also be seen in Fig. 2b, where both ion and electron densities are depicted. The space charge extension clearly expands towards the plasma with lower values of $\theta$, while the plasma density at the non-neutral area entrance shows an important diminution, from about $0.40 \times n_{0}$ at $90^{\circ}$ to less than $0.05 \times n_{0}$ below $10^{\circ}$.

Fig. 3a shows the spatial variation of the electric potential for the entire plasma and for increasing values of $\phi_{w}$ in the case $\theta=15^{\circ}$. As expected, the situations for $\phi_{w}<0$ and $\phi_{w}>0$ are completely symmetrical: when $\phi_{w}<0$ (resp. $>0$ ), the vast majority of the applied voltage drops within the sheath at the left (resp. right wall). Even for large

positive $\phi_{w}$, the potential variation between the left wall and the center of the plasma keeps a negative value, that is why an ion current arises at both electrodes for any $\phi_{w}$ in such low collisionality case. Fig. 3b depicts the velocity profiles at the left wall for the same values of $\phi_{w}$ as in Fig. 3a. For $\phi_{w} \geq 0$, the ion velocity profiles are very similar and $v_{i x}$ is not supersonic: for such wall potentials, there is no Debye sheath (note that for $\phi_{w}=0$ the ion velocity at the wall $v_{i x} \rightarrow 0.98 c_{s}$, which is very close to the arbitrary critical value used in this paper for defining the sheath entrance). However, for $\phi_{w}<-10 \mathrm{~V}$, the ion velocity flow is clearly supersonic at the electrode vicinity. Furthermore, $\left|v_{i x}\right|$ increases with $\left|\phi_{w}\right|$ and the Debye sheath extent $s$ is redefined with respect to the point where $v_{i x}$ reaches $-c_{s}$. As shown in Fig. 3b, s increases with $\left|\phi_{w}\right|$ as expected.

The absence of the Debye sheath in floating wall conditions is due to the large potential drop existing in the Chodura sheath for such small incidence of the magnetic field. As explained above, it has been shown that ions enter the Chodura region with a velocity 

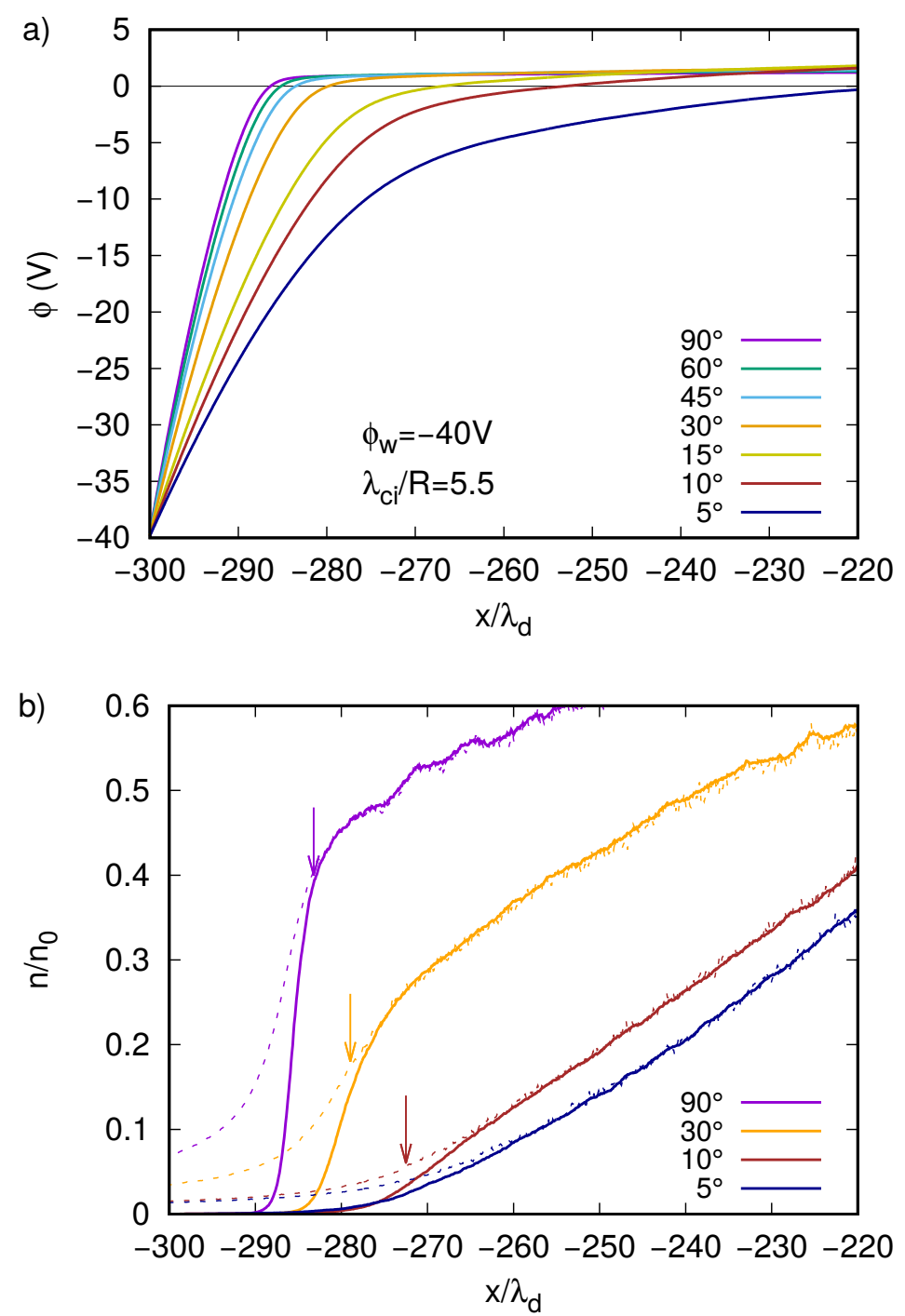

FIG. 2. a) Spatial variation of the electric potential for different angles of incidence of the magnetic field in the case of a low collision rate $\left(\lambda_{c i} / R=5.5\right)$ and for a wall potential of $\phi_{w}=-40 \mathrm{~V}$. b) Ion (dashed line) and electron (continuous line) density at the vicinity of the left wall for different $B$ inclination and $\phi_{w}=-40 \mathrm{~V}$. The arrows mark the sheath entrance location using the Bohm criterion.

of $c_{s} \sin \theta$ in the direction perpendicular to the wall and become supersonic at the Debye entrance in cases with large enough incidence for the Debye sheath to form. Assuming for the sake of simplicity that there is no ionization in these regions of the plasma, one can write that: 

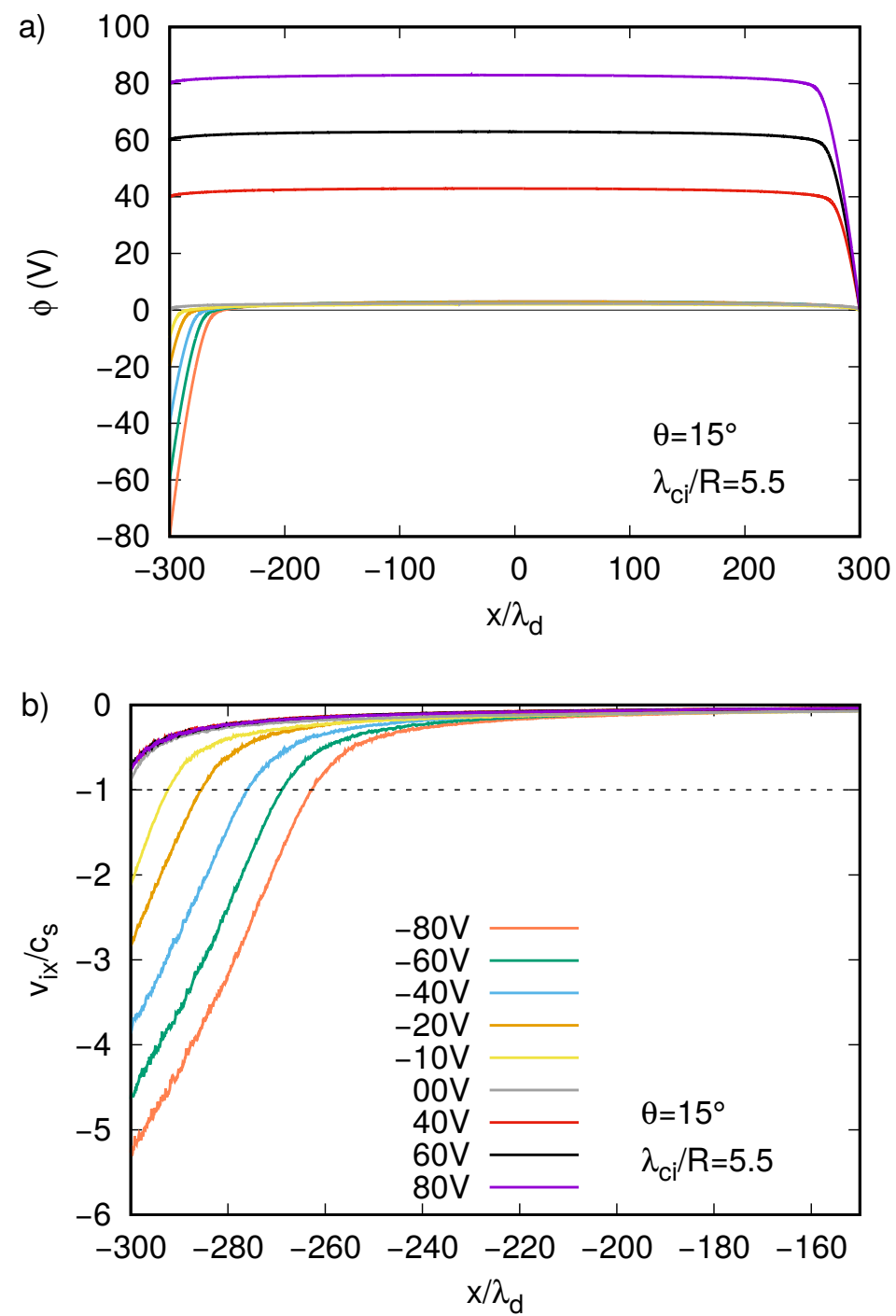

FIG. 3. a) Spatial variation of the electric potential for an angle of incidence of $15^{\circ}$ and $\lambda_{c i} / R=5.5$ and for increasing wall potentials. b) Ion average velocity profiles normalized to the acoustic velocity $c_{s}$ for increasing wall potentials. The intercept between the dashed line and the velocity profiles gives the sheath entrance location.

$$
n_{s} c_{s}=n_{c h} c_{s} \sin \theta
$$

with $n_{s}$ and $n_{c h}$ the ion density at the Debye and Chodura sheath entrances respectively. If collisions can be neglected for electrons, their density $n_{e}$ is expected to follow the Boltzmann relation. The Chodura sheath being quasi-neutral, it is straightforward to calculate the potential drop in the Chodura sheath, $\Delta \phi_{c s}$ as: 


$$
\frac{e \Delta \phi_{c s}}{k_{b} T_{e}}=\ln (\sin \theta)
$$

which is a negative quantity. The amplitude of $\Delta \phi_{c s}$ increases with decreasing the magnetic field incidence. With similar arguments, the total potential drop $\Delta \phi_{t}$ between the Chodura sheath entrance and the wall can be calculated as independent of the magnetic field incidence as:

$$
\frac{e \Delta \phi_{t}}{k_{b} T_{e}}=\frac{1}{2} \ln \left[2 \pi \frac{m}{M}\left(1+\frac{T_{i}}{T_{e}}\right)\right]
$$

which is also a negative value, $m$ and $M$ are the electron and ion mass respectively and $T_{i}$ the ion temperature.

It exists then, as pointed out by Stangeby ${ }^{19}$, a critical angle $\theta^{*}$ below which the potential drop within the Debye sheath, $\Delta \phi_{d}=\Delta \phi_{t}-\Delta \phi_{c s}$, goes to zero. According to Eq. (4) and Eq. (5), it comes, in floating wall conditions:

$$
\sin \theta_{0}^{*}=\left[2 \pi \frac{m}{M}\left(1+\frac{T_{i}}{T_{e}}\right)\right]^{1 / 2} .
$$

With the electron and ion temperature and the mass ratio used in the simulations, $\theta_{0}^{*} \simeq 9.12^{\circ}$. However, according to our results, it comes that the Debye sheath disappears for $\theta \leq 15^{\circ}$ in floating wall conditions, which is a bit larger than the expected theoretical value as we already discussed elsewhere ${ }^{21}$ and which can be attributed to either collisions (neglected in the fluid model) or to kinetic effects (to derive expression (5) electrons are assumed to follow the field line up to the wall).

The situation is different in non-floating conditions. When $\left|\phi_{w}\right|>\left|\Delta \phi_{t}\right|$, the potential drop within the Debye sheath is given by $\Delta \phi_{d}=\phi_{w}-\frac{k_{b} T_{e}}{e} \ln (\sin \theta)$, which results in a smaller critical angle $\theta^{*}$. With our plasma parameters, $\Delta \phi_{t}$ is expected to be approximately $3.68 \mathrm{~V}$ and for an incidence of $\theta=15^{\circ}$, the potential drop would be of the order of $2.7 \mathrm{~V}$ within the Chodura sheath using Eq. (4). Thus when $\left|\phi_{w}\right|>>3.68 \mathrm{~V}$, it is reasonable to consider that the vast majority of the applied voltage drops in the Debye sheath even for such a relatively small incidence.

Fig. 4a shows the variation of the sheath size $s$ with respect to the wall potential $\phi_{w}$ for different incidences between $90^{\circ}$ and $15^{\circ}$ in the low collisionality case. When $\phi_{w} \geq 0$, the situation at the left wall is similar to that with floating wall conditions and the sheath 

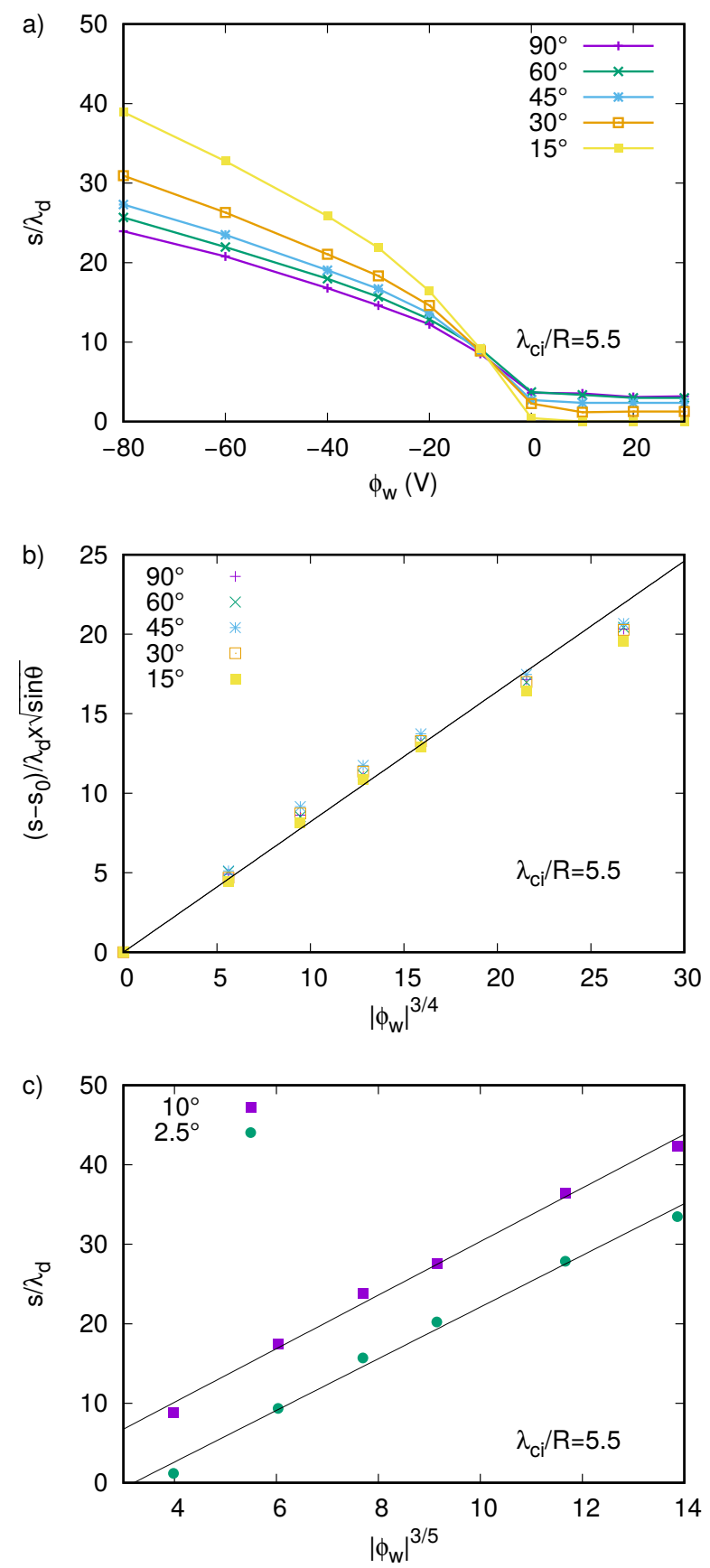

FIG. 4. a) Variation of the sheath size $s$ with respect to the wall potential $\phi_{w}$ for different incidences of the magnetic field and $\lambda_{c i} / R=5.5$. b) Variation of the normalized sheath size with respect to the $3 / 4$ power of the wall potential for the same incidences and collisionality than in a). c) Variation of the sheath size $s$ with respect to the $3 / 5$ power of $\phi_{w}$ for grazing incidences. The continuous lines in the figure are used as a guide to the eye. 
size decreases with the angle of incidence, because the part of the potential drop within the Chodura layer increases with decreasing $\theta$, as explained previously. This inevitably reduces the potential drop inside the Debye sheath, as well as the sheath size, until it eventually disappears.

For negative wall potentials, the sheath size increases with both increasing $\left|\phi_{w}\right|$ and decreasing values of the magnetic field incidence, which is consistent with Fig. 2. This behavior can be ascribed to the density reduction at the Debye sheath entrance when $\theta$ decreases, because of the drop of density in the Chodura sheath, which scales with $\sin \theta$. It is possible to derive the Child-Langmuir law in a very simple and straightforward way, as already proposed in reference ${ }^{37}$, by assuming the ion continuity equation between the Chodura layer entrance and the Debye sheath as:

$$
n_{s h} v_{s h}=n_{c h} c_{s} \sin \theta
$$

with $n_{s h}$ and $v_{s h}$ the ion density and velocity inside the sheath respectively, which are both assumed as constant. This is a strong assumption, which is only used to recover the Child-Langmuir law in a few steps. If $\omega_{c i} \ll \omega_{p i}$, one can argue that most of the ion motion is perpendicular to the wall in the sheath region, therefore it is possible to write the conservation of the ion energy as:

$$
\frac{1}{2} M v_{s h}^{2}+e \phi_{w}=\frac{1}{2} M c_{s}^{2}+e \phi_{s} .
$$

The potential at the sheath entrance $\phi_{s}$ can be been taken as a reference ie. $\phi_{s}=0$. Assuming that the electron density $n_{e} \simeq 0$ in the sheath, the Poisson equation can be solved as:

$$
\frac{\phi_{w}}{s^{2}} \simeq-\frac{n_{s h} e}{\epsilon_{0}}
$$

Considering that $v_{s h} \gg c_{s}, T_{i} \simeq T_{e}$, and combining Eq. (7), (8) and (9), it comes:

$$
\frac{s}{\lambda_{d}} \simeq \frac{1}{\sqrt{\sin \theta}}\left(\frac{-e \phi_{w}}{T_{e}}\right)^{3 / 4}
$$

which is equivalent to Eq. (2), when $J_{i} \propto \sin \theta$. According to Eq. (10), plotting $s \sqrt{\sin \theta}$ vs. $\phi_{w}$ would rescale the $s=f\left(\phi_{w}\right)$ characteristics on a single curve. As shown in Fig. 4b, the Child-Langmuir law with a $3 / 4$ exponent is in a relatively good agreement with the 
simulations results for the sheath size as long as the $\sin \theta$ variation of the density at the sheath entrance is taken into account. A similar conclusion was raised by other authors in a $2 \mathrm{D}$ study $^{38}$. We should point out that the best average exponent for the $s$ vs. $\left|\phi_{w}\right|$ previous characteristics is $\simeq 0.7$, but our goal was to determine the best exponent according to the main existing models. Note also that the sheath size in floating wall conditions $s_{0}$ was subtracted to the sheath size $s$ in Fig. $4 \mathrm{~b}$, because the variation of $s_{0}$ with $\theta$ is opposite, as detailed previously and in reference ${ }^{38}$ as well.

For grazing incidences, below $15^{\circ}$, another key aspect must be highlighted, which is the absence of Chodura sheath, even in the low collisional case. As explained in the introduction of this paper, collisions can occur at one ion Larmor radius distance from the wall for $\theta<\arcsin \frac{\lambda_{c i}}{R}$, ie. $\theta<10.47^{\circ}$ for the plasma parameters used in our simulations. For such incidences, the plasma density at the Debye sheath does not vary with $\sin \theta$ anymore, and the ion current $J_{i}$ crossing the sheath depends on collisions: both $J_{i}$ and the sheath size decrease with increasing collisionality.

Fig. 4c shows the sheath size variation vs. a $3 / 5$ power of the wall potential, which is the exponent law usually adopted to model the sheath size in the intermediate collisionality case, for two grazing incidences of $2.5^{\circ}$ and $10^{\circ}$. There is again a very good agreement between the Child-Langmuir law and the simulations results; the sheath size also decreases with decreasing $\theta$ (ie. decreasing the collisionality), as expected ${ }^{4}$. The fact that the exponent corresponding to the intermediate collisionality fits very well the calculated sheath size is due to the moderate number of collisions which may arise within the sheath region. This

number can be evaluated by using the ratio $\frac{\lambda_{c i} \sin \theta}{\lambda_{d}}$, as about 4.94 for an incidence of $\theta=2.5^{\circ}$ : with $s$ in the range $10-40 \lambda_{d}$, a few collisions are expected when ions are crossing of the sheath, and that is why the ion velocity $v_{i x} \gg v_{t i}$ in this plasma region.

\section{REGIME OF INTERMEDIATE AND LARGE COLLISIONALITY}

When $\lambda_{c i} / R<1$, ions undergo a sufficient number of collisions to undermine the magnetic field effect. In a first approximation, the ion motion can be assumed to be directed towards both walls independently of the field incidence $\theta$. For electrons, however, the fact that $\lambda_{c e} / r>1$ preserves the anisotropy of their velocity field, because they are forced to follow the magnetic line up to the wall. 
Fig. 5a shows the spatial variation of the electric potential for $\phi_{w}=-40 \mathrm{~V}$ for decreasing magnetic field incidences in the case $\lambda_{c i} / R=0.55$. We may distinguish 3 ranges of incidences. Firstly, for $\theta>15^{\circ}$, the potential profiles are almost identical, with a large potential drop across the left sheath as expected. Fig. 5b depicts the corresponding ion velocity profiles along the $x$ axis, all reaching sonic velocity at a similar abscissa, leading to similar sheath sizes as shown in Fig. 5c. Once again, an exponent of $3 / 5$ seems to fit quite well the variation of the sheath size vs. $\left|\phi_{w}\right|$. The number of collisions undergone by the ions crossing the sheath can be evaluated with the ratio $\frac{\lambda_{c i}}{\lambda_{d}}=11.3$ (independent of $\theta$ since the ion motion is mainly along $x$ ), which would lead, as previously, to a moderate collisionality of the ions within the sheath.

Secondly, when the incidence of the magnetic field is decreased below $15^{\circ}$, the potential profiles begin to change as depicted in Fig. 5a. For $\theta=10^{\circ}$, the potential mostly keeps a negative and continuously increasing value inside the plasma, so that the left sheath is only able to screen partially the applied voltage. The variation of the sheath size for this specific incidence is also shown in Fig. 5c and follows very well the Child-Langmuir law with a 3/5 exponent.

Finally, for $\theta<5^{\circ}$, the ion flow keeps subsonic values at the left electrode suggesting the absence of a Debye sheath, and for the extreme grazing incidence of $\theta=0.5^{\circ}$, a potential drop of the order of $\left|\phi_{w}\right|$ appears close to the right wall instead of the left one. Fig. 6a depicts the potential profiles in the entire simulated plasma for $\theta=3.5^{\circ}, \lambda_{c i} / R=0.55$, and increasing wall potentials, from $-80 \mathrm{~V}$ to $80 \mathrm{~V}$. It is worth comparing the latter figure with what was presented in Fig. 3a, as it is the mirror situation; a large potential drop occurs at the left wall but for positive $\phi_{w}$. The corresponding ion and electron densities are shown in Fig. $6 \mathrm{~b}$ for $\phi_{w}>0$ only. For $\phi_{w}=0$ and $10 \mathrm{~V}$ the space charge at the vicinity of the left electrode is negligible. However, when $\phi_{w} \geq 20 \mathrm{~V}$, an electro-negative space charge arises, which extends over tens of $\lambda_{d}$, just like a classical Debye sheath. This negative space charge screens the positive wall voltage from the plasma and that is why there is a strong potential drop in this region.

The transition from the usual regime to this opposite one can be seen on the particle flow at the left wall as depicted in Fig. 7. When the incidence of the magnetic field is larger than $\theta>10^{\circ}$, the ion flow at the left wall (see Fig. 7a) is around $\simeq 0.15 \times n_{0} c_{s}$ for negative wall potentials and $\simeq 0.12 \times n_{0} c_{s}$ for $\phi_{w}>0$ and it does not vary much with $\theta$ (because 

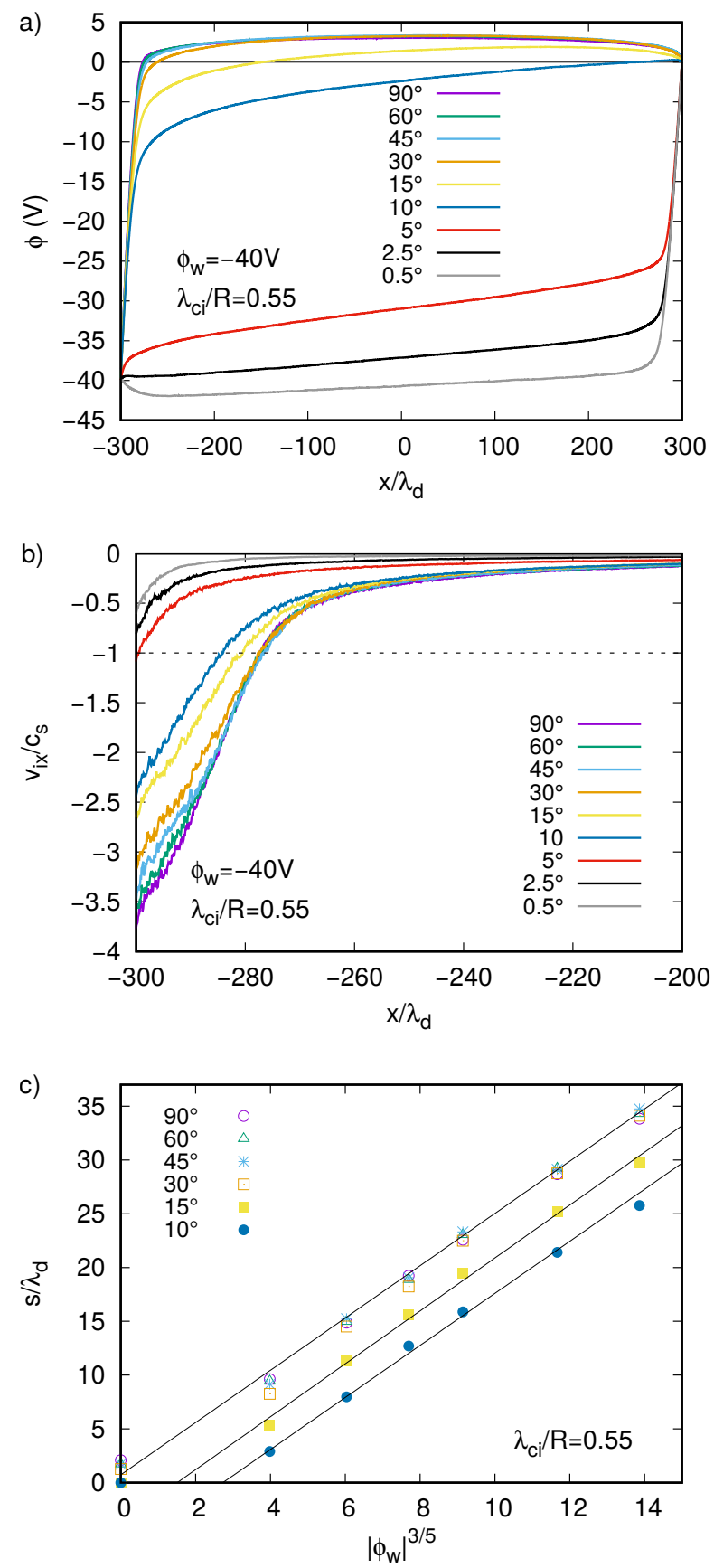

FIG. 5. a) Spatial variation of the electric potential for different angles of incidence of the magnetic field in the case of an intermediate ion collision rate $\left(\lambda_{c i} / R=0.55\right)$ and for a wall potential of $\phi_{w}=-40 \mathrm{~V}$. b) Corresponding ion velocity profiles. c) Variation of the sheath size $s$ with respect to the $3 / 5$ power of the wall potential $\phi_{w}$ for various incidences of the magnetic field. The continuous lines in the figure are used as a guide to the eye. 

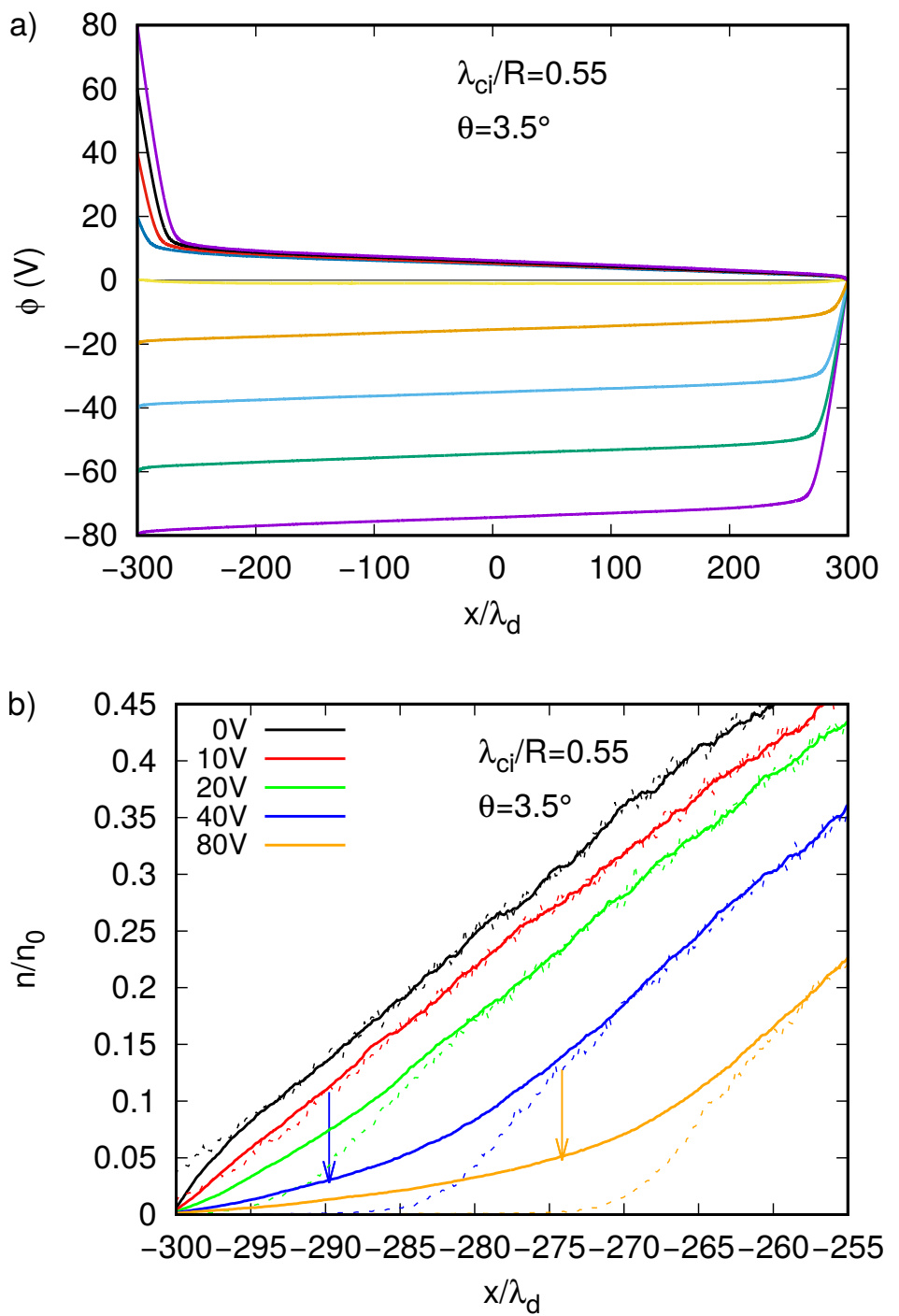

FIG. 6. a) Spatial variation of the electric potential for an incidence of $3.5^{\circ}$, an intermediate ion collisionality $\left(\lambda_{c i} / R=0.55\right)$, and various wall potentials. b) Ion (dashed line) and electron (continuous line) density at the vicinity of the left wall for an incidence of $3.5^{\circ}$ and increasing positive values of $\phi_{w}$. The arrows mark the entrance of the inverse sheath deduced from the ion sonic point location.

ions are demagnetized by collisions). The electron flow however (see Fig. 7b) vanishes for negative wall potentials, so that electrons entirely escape through the right electrode (the mirror situation arises for positive wall potentials). When $\theta<10^{\circ}$ though, the configuration is opposite, with the electro-negative charge at the left wall which reflects the ion flow for $\phi_{w}>0$. In this case of positive potential at the left wall, ions mainly escape through the 
right electrode, unlike electrons whose flow is a bit larger on the left electrode but remains partially on the right one.

For such grazing incidences of the magnetic field, electrons with a collision rate lower than their cyclotron frequency, have a lower mobility in the $x$ direction than their positive counterpart due to magnetic field effects. The electro-negative space charge is then there to prevent the total ion flow from overcoming the total electron flow at both walls and to preserve the plasma quasi-neutrality. We already emphasized in our previous study that such a scenario could occur for a certain range of both incidence and collisionality. ${ }^{21,35}$ It has also been shown by several authors for different plasma conditions: in the presence of strong electron emission at the surface, a double sheath ${ }^{39}$, or an inverse sheath ${ }^{40}$, can form in order to shield the positive wall potential; a similar phenomenon was described when the ratio of surface collection of ions over that of electrons is such that ions leave the plasma to one surface while electrons are lost to a different one ${ }^{41}$.

In order to determine a sheath size in such unusual situations, it is worth modeling the quasi-neutral pre-sheath in order to determine if a critical velocity appears in the fluid equations, just like the ion sonic point for the Debye sheaths, which could be used as a reliable sheath entrance condition.

In this case of large collisionality for ions, ie. $\lambda_{c i}<R$, we can neglect in a first approximation the magnetic field contribution in the ion fluid equations since collisions overcome the magnetic order ${ }^{21}$. The electric field being oriented along the $x$ axis, there is only a net drift in this direction and velocities in the $y$ and $z$ directions are such that $v_{i z} / v_{t i} \simeq v_{i y} / v_{t i} \ll 1$. In the steady state, the fluid equation of momentum conservation on the $x$ axis, denoting the derivative in $x$ by a prime symbol, reads for ions:

$$
M v_{i x} v_{i x}^{\prime}=-e \phi^{\prime}-T_{i} \frac{n_{i}^{\prime}}{n_{i}}-M \nu_{i} v_{i x}
$$

The continuity equation is:

$$
\left(n_{i} v_{i x}\right)^{\prime}=\left(n_{e} v_{e x}\right)^{\prime}=S,
$$

with $S$ a source term kept constant in our simulations. In the pre-sheath, as the quasineutrality stands and $n_{i} \simeq n_{e} \simeq n$, we can write that: 

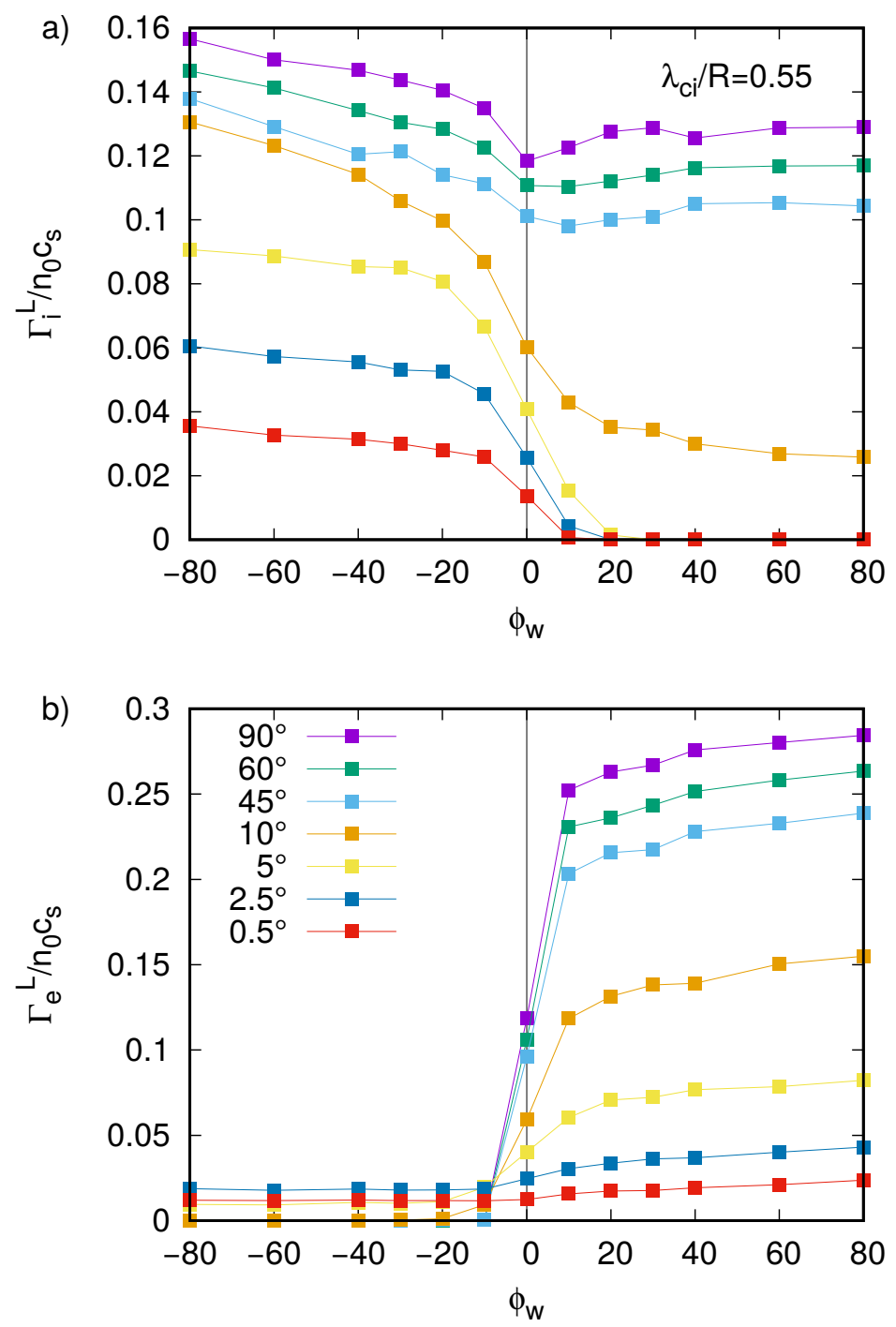

FIG. 7. a) Variation of the ion flow at the left wall $\Gamma_{i}^{l}$ vs. the wall potential for different incidences of the magnetic field (given in the key to symbols of Fig. 7b). b) Variation of the electron flow at the left wall $\Gamma_{e}^{l}$ with respect to $\phi_{w}$.

$$
n v_{i x}=n v_{e x}+\gamma
$$

In floating wall conditions $n v_{i x}=n v_{e x}$, ie. $\gamma=0$, because both electrons and ions fluxes at the walls are balanced in order to preserve the quasi-neutrality of the plasma. When a voltage is applied between both plates however, a current is circulating through the plasma column and in case of a positive value of $\phi_{w}, \gamma>0$.

The fluid equations of momentum conservation for electrons are: 


$$
\begin{gathered}
m v_{e x} v_{e x}^{\prime}=e \phi^{\prime}-e v_{e y} B \cos \theta-T_{e} \frac{n_{e}^{\prime}}{n_{e}}-m \nu_{e} v_{e x} \\
m v_{e x} v_{e y}^{\prime}=e v_{e x} B \cos \theta-e v_{e z} B \sin \theta-m \nu_{e} v_{e y} \\
m v_{e x} v_{e z}^{\prime}=e v_{e y} B \sin \theta-m \nu_{e} v_{e z}
\end{gathered}
$$

Neglecting as usual inertia terms for electrons and using Eq.(15) and Eq.(16), yields:

$$
v_{e y}=\frac{\omega_{c e} \cos \theta}{\nu_{e}+\frac{\omega_{c e}^{2}}{\nu_{e}} \sin ^{2} \theta} v_{e x}
$$

Substituting Eq. (17) into Eq. (14), we have for the electric potential:

$$
e \phi^{\prime}=T_{e} \frac{n^{\prime}}{n}+m \nu_{e \theta} v_{e x}
$$

with $\nu_{e \theta}=\nu_{e} \frac{\nu_{e}^{2}+\omega_{c e}^{2}}{\nu_{e}^{2}+\omega_{c e}^{2} \sin ^{2} \theta}$. When the collisional term in Eq. (18) is small with respect to the pressure term, the Boltzmann relation is recovered. But when it can not be neglected, the electron collisionality increases when the magnetic field incidence decreases. Using Eq.(11), (12) and Eq.(18), it comes:

$$
\frac{n^{\prime}}{n}=\frac{-\left(\nu_{i}+\nu_{s}\right) v_{i x}-\mu \nu_{e \theta} v_{e x}}{c_{s}^{2}-v_{i x}^{2}},
$$

where $\mu=m / M$ and $\nu_{s}$ is a ionization frequency defined as $\nu_{s}=S / n$.

The derivative of the density must keep a positive value on the left wall. Therefore for any ion velocity such as $\left|v_{i x}\right|<c_{s}$, the numerator of the right member of Eq. (19) must be positive. $v_{e x}$ is necessarily negative, so that the condition on the numerator sign is fulfilled for any negative $v_{i x}$. However, in situations where $v_{i x}>0$, which can occur for large $e \phi_{w}>k_{b} T_{i}$, grazing incidences and sufficient collisionality, one can write, using Eq. (13), the previous condition on the numerator as:

$$
\Gamma_{i}(x)<\frac{\mu \nu_{e \theta}}{\nu_{i}+\nu_{s}+\mu \nu_{e \theta}} \times \gamma=g(\nu, \theta) \times \gamma .
$$

For $x \in[-L / 2,0]$, the ion flux is expected to be positive and strictly increasing with $x$, therefore condition (20) is always met as long as $\Gamma_{i}(0)<g(\nu, \theta) \times \gamma$. Assuming that $\Gamma_{i}(-L / 2) \simeq 0$, it comes that $\Gamma_{i}(0) \simeq \frac{1}{2}\left(\Gamma_{e}(L / 2)+\gamma\right) \simeq \frac{\gamma}{2}$ (the main electron current 
circulates through the left electrode for large positive $\phi_{w}$ ). Therefore an acceptable estimate of condition (20) would give $g(\nu, \theta) \geq \frac{1}{2}$. Using $\nu_{i, e}=\frac{v_{t i, e}}{\lambda_{c i, e}}, \lambda_{c i}=0.55 R$, and assuming $\nu_{s}<<\nu_{i}$, we have $g(\nu, \theta)=0.50,0.59,0.84,0.96$ for $\theta=6^{\circ}, 5^{\circ}, 2.5^{\circ}, 1^{\circ}$ respectively. Then for grazing incidences of the magnetic field, ie. below $6^{\circ}$, the numerator of expression (19) is always positive, thanks to the electron collision term, in case of a positive ion velocity. Finally the quasi-neutrality fails for $v_{i x}=c_{s}$, where the plasma approximation $n_{i} \simeq n_{e}$ does not stand anymore and the derivative of the density diverges. The ion sonic point can be used to determine the electro-negative sheath entrance, but in this case, it is not the usual ongoing sheath velocity, but the outgoing one instead.

Fig. 8a shows the ion velocity profiles close to the left wall, where the electro-negative sheath arises, for $\theta=3.5^{\circ}, \lambda_{c i} / R=0.55$ and different values of $\phi_{w}$. The ion average velocity becomes progressively positive, when $\phi_{w}$ is increased, and for $\phi_{w} \geq 40 \mathrm{~V}$, there is no more ion current at the collecting surface. The non monotonic velocity characteristics observed for such an amplitude of $\phi_{w}$ is due to different contributions to the average velocity: ions born at rest in the sheath are strongly accelerated towards the plasma, leading to a positive and increasing average velocity at the wall vicinity, where the space electric field is large; on the other side of the sheath, there are ions with positive and negative velocities, coming from the plasma and reflected by the sheath, so that the average velocity decreases. These different contributions to the average ion velocity can also be seen in the example of phasespace plot shown in Fig. 9a for a grazing incidence of $5^{\circ}$ and a wall potential of $\phi_{w}=80 \mathrm{~V}$. A phase-space plot for a larger incidence of $60^{\circ}$ and $\phi_{w}=-80 \mathrm{~V}$, where a strong ion current flows towards the left collecting surface, is also shown in Fig. 9b for comparison.

Fig. 8b shows the sheath size deduced from the position where the ion velocity reaches $v_{i x}=+c_{s}$. The sheath size increases with increasingly grazing incidence of the magnetic field (decreasing $\theta$ ), because the electron mobility towards the wall diminishes. Moreover, the minimum wall potential required to make ions reach $c_{s}$ in order to fulfill the inverse sheath criterion decreases with $\theta$ for the same reason. Finally, the variation of this inverse sheath follows the Child-Langmuir law with a $2 / 3$ exponent. There is a particularly good agreement for $\theta=0.5^{\circ}$. Here the $2 / 3$ power dependence of the surface potential for the sheath size is due to the electron collisionality in the sheath region, because they are the crossing species instead of ions. With a similar argument than previously, the number of electrons vs. neutrals collisions in the sheath can be evaluated using the ratio $\frac{\lambda_{c e} \sin \theta}{\lambda_{d}}$, which 

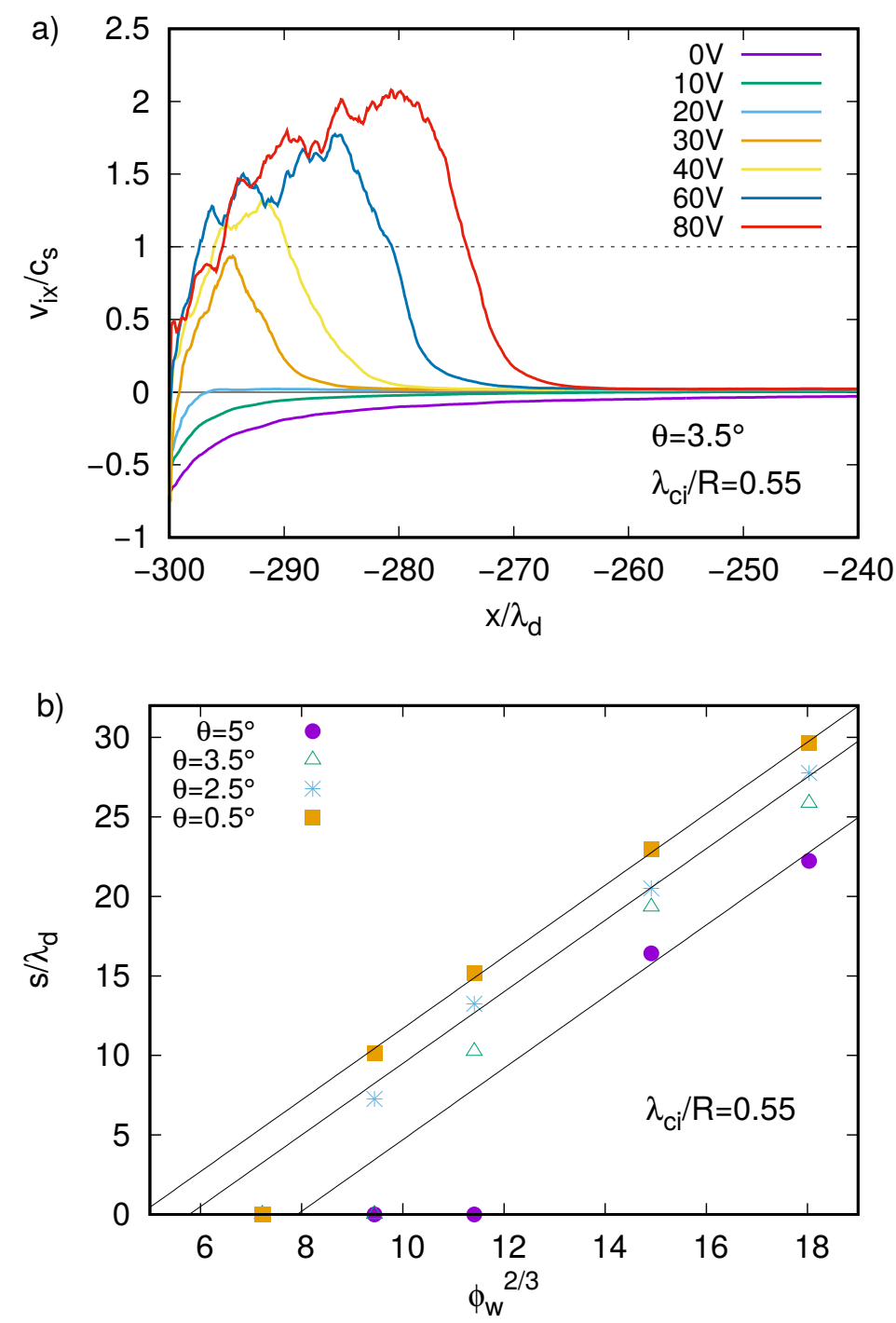

FIG. 8. a) Ion velocity profiles for increasing values of the wall potential $\phi_{w}$, for $\theta=3.5^{\circ}$ and $\lambda_{c i} / R=0.55$. b) Variation of the sheath size $s$ with respect to the $2 / 3$ power of the wall potential for grazing incidences only and $\lambda_{c i} / R=0.55$. The continuous lines in the figure are used as a guide to the eye.

is of the order of 3.95 and 1.98 for $\theta=5$ and $2.5^{\circ}$ respectively. For incidences such that $\theta<\arctan \frac{r}{\lambda_{c e}}$, the characteristic ratio is rather $\frac{r \cos \theta}{\lambda_{d}} \simeq \frac{r}{\lambda_{d}} \simeq 0.90$, which makes the sheath highly collisional for electrons.

When the collisionality is further increased up to $\lambda_{c i} / R=0.10$ and $\lambda_{c e} / r=10$, the same conclusion can be raised than for the previous case with $\lambda_{c i} / R=0.55$ for incidences $\theta \geq 30^{\circ}$ as depicted in Fig. 10a and b. However for grazing incidences, an intermediate situation 

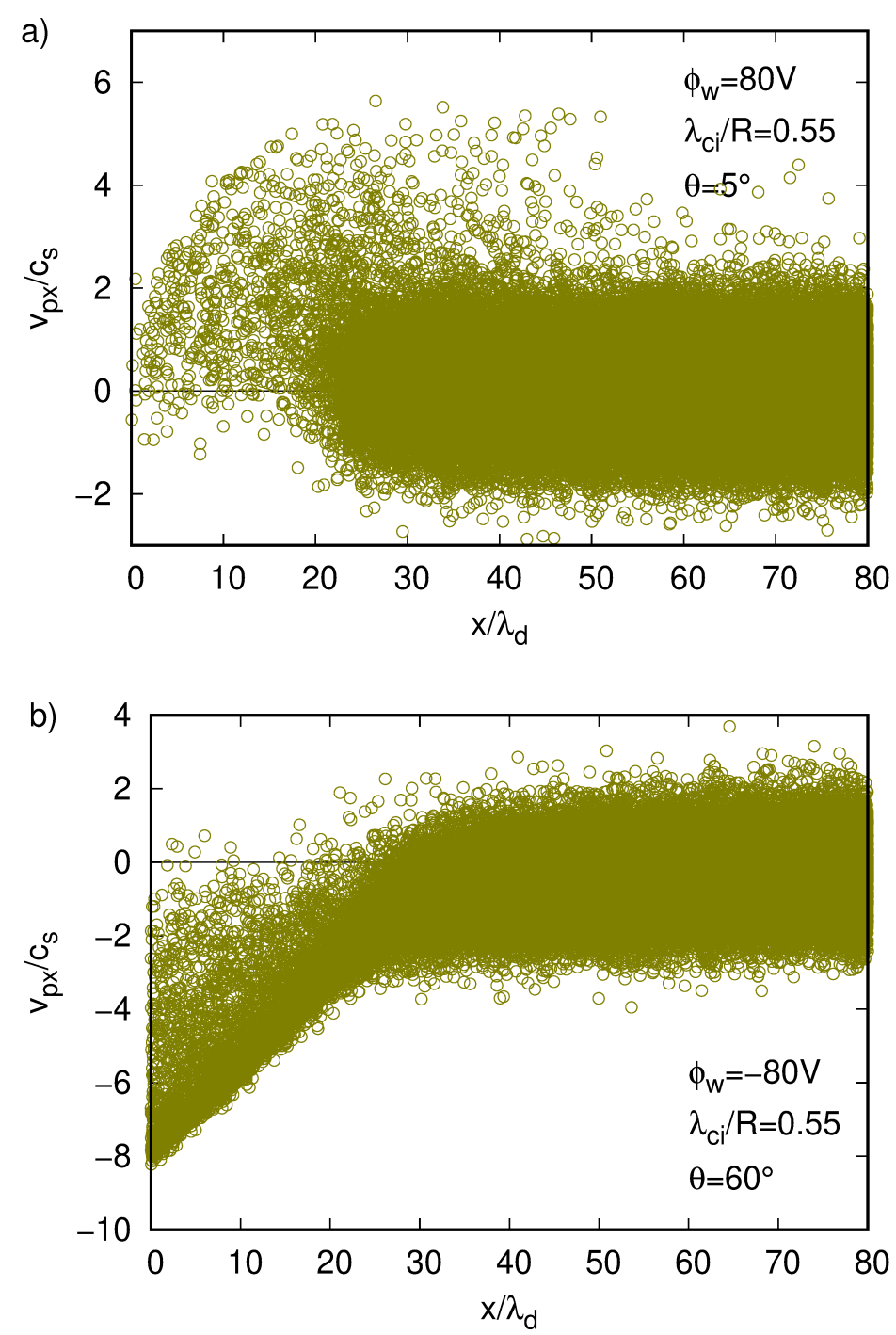

FIG. 9. a) Phase-space plot of ions close to the left wall for $\theta=5^{\circ}$, a wall potential of $\phi_{w}=80 \mathrm{~V}$ and a collisionality of $\lambda_{c i} / R=0.55$. b) Phase-space plot of ions for the same collisionality than in a), a wall potential of $\phi_{w}=-80 \mathrm{~V}$ and an incidence of $\theta=60^{\circ}$.

arises, where a large potential drop occurs at both walls (see Fig. 10a) as well as within the plasma: there is no total screening of the applied voltage by a single sheath as described in the case of low and moderate ion collisionalities. The ion velocity reaches the sonic point down to incidences of $2.5^{\circ}$, where $s$ is only about $7.8 \lambda_{d}$ for $\phi_{w}=-80 \mathrm{~V}$, instead of tens of $\lambda_{d}$ for the lowest collisionality (see eg. Fig. 4c). But $v_{i x}$ keeps modest values at the wall vicinity, barely exceeding $c_{s}$, whereas for lower collisionality, it can reach $5 \times c_{s}$. On the right wall, for a negative left wall potential, there is, as mentioned above, another important 
potential drop, which corresponds to an electro-negative space charge. However, the ion velocity does not reach the sonic point (not shown), so that we can not conclude regarding the existence of an inverse sheath in this highly collisional case. Note that when the ratio $\lambda_{c e} / r$ diminishes down to 1 and below, the electron mobility in the $x$ direction towards the wall increases and the situation would eventually lead to a non-magnetized system, with highly collisional sheaths. We have already explored this tendency in our previous study ${ }^{21}$. As a matter of fact, the sheath size has been plotted with respect to a $2 / 3$ power of the wall potential in Fig. 10c, which seems to give quite linear variations, despite a relatively important noise. A 2/3 exponent is usually employed in the Child-Langmuir law, in case of collisional sheaths, where the ion average velocity is smaller than the thermal velocity as explained in the introduction of this paper. The ratio $\frac{\lambda_{c i}}{\lambda_{d}}$ is about 2.27 , which makes ion vs. neutral collisions within the sheath region highly probable. Compared to the study of Sheridan and Goree ${ }^{4}$ (see their Fig. 6), the latter ratio would place this specific case in the collisional regime but near the frontier with the transition one.

\section{CONCLUSION}

We have investigated by means of PIC simulations the behavior of a 1D bounded plasma under an external oblique magnetic field, when a constant voltage is applied between the two electrodes. Collisions of the charged particles with neutrals were taken into account using a simple hard-sphere model and a Monte Carlo standard procedure. Different meanfree-path to Larmor ratios were used in order to explore the effects of collisions onto the plasma wall transition, and more particularly onto the sheath size. The electron meanfree-path was always larger than the electron Larmor radius, so that electrons can travel along the field line for several gyroperiods without undergoing a collision. We have explored however different regimes for the ion collisionality. For the lowest collision rate, $\lambda_{c i} / R>1$, we have shown that the sheath size $s$ scales with $\sin \theta: s$ increases with decreasing the angle of incidence of the magnetic field $\theta$. It was also shown that $s$ follows very well the ChildLangmuir law with a $3 / 4$ exponent of the wall potential $\phi_{w}$. When the field incidence is such that $\theta<\arcsin \frac{R}{\lambda_{c i}}$ though, the scaling of $s$ with $\sin \theta$ no longer exists because of the merging of the Chodura sheath with the collisional pre-sheath. For such grazing incidences, a Child-Langmuir law with a 3/5 exponent of the wall potential is a better fit of the sheath 

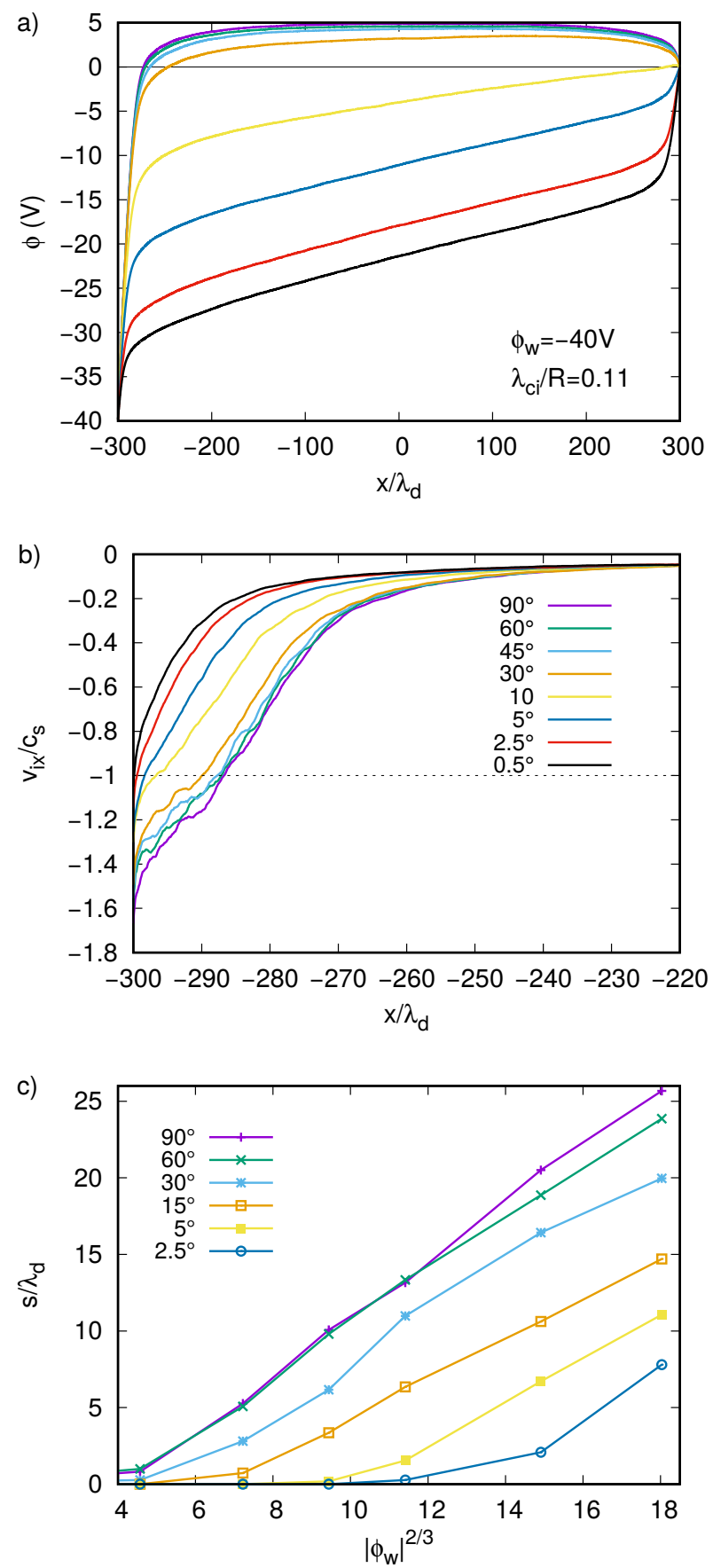

FIG. 10. a) Spatial variation of the electric potential for different angles of incidence of the magnetic field in the case of a high ion collision rate $\left(\lambda_{c i} / R=0.10\right)$ and for a wall potential of $\phi_{w}=-40 \mathrm{~V}$ b) Corresponding ion velocity profiles. c) Variation of the sheath size $s$ with respect to the wall potential $\phi_{w}$ for various incidences of the magnetic field.

size. When the ion collisionality is increased to a typical ratio of $\lambda_{c i} / R \simeq 0.5$, as long 
as incidences are larger than $15^{\circ}$, the sheath size does not vary with $\theta$, because ions are demagnetized by collisions and travel towards the wall by a random walk, whose step is the ion mean-free-path. $s$ can still be modeled by a Child-Langmuir law with a $3 / 5$ exponent of the wall potential, thanks to a moderate number of collisions within the Debye sheath. For the same collisionality but grazing incidences however, an interesting phenomenon occurs: for positive wall potentials, an electro-negative sheath arises, whose spatial extension is, like a classical Debye sheath, of the order of tens of $\lambda_{d}$. We have demonstrated that the ion velocity at the exit of this inverted sheath is the acoustic ion velocity, which can be used as a reliable criterion to determine the sheath size. This electro-negative sheath arises when ions migrate towards the wall faster than electrons, which are forced to follow the field line up to the wall. For larger collisionalities, ie. $\lambda_{c i} / R<<1$, intermediate situations take place, where a classical Debye sheath can build up at one wall, while an electro-negative charge region to the other, although the ion velocity does not reach the sonic point. Finally in our simulations, a classical Child-Langmuir law with typical exponents of $3 / 4,3 / 5$ and $2 / 3$ allows an acceptable calculation of the sheath size depending on the collisionality. We would like to point out however that we did not extract the exact exponents from the simulations but tried to find the best fitted one according to existing models, and that choosing between $2 / 3$ and $4 / 5$ was sometimes arduous in collisional cases.

Finally, the effect of the magnetic field is twofold: for large incidences and low collisional rates, it reduces the ion flow entering the sheath, leading to a scaling of $s$ with $\theta$; for grazing incidences, it delays the electron flow towards the wall, which can lead, for a specific collisionality range, to an inverse sheath, already evidenced by other authors but for quite different plasma conditions. We already derived in reference ${ }^{21}$ a formula which gives the critical magnetic field incidence $\theta_{p}$ at which the polarity of the ambipolar field within the collisional pre-sheath is expected to change from negative to positive with respect to the mean-free-paths to the Larmor radii ratios. This regime where ions need to be pushed back into the plasma in the quasi-neutral region is favorable to the inversion of the space charge polarity within the sheath. Assuming for the sake of simplicity that the incidence is such that $\theta \simeq 0$ and $k_{b} T_{i}=k_{b} T_{e}=2 \mathrm{eV}$, we have also showed ${ }^{35}$ that the ambipolar field inversion is expected for demagnetized ions (ie. $\lambda_{c i}<R$ ) when $\lambda_{c i} \lambda_{c e}>r^{2} \sqrt{M / m}$. With a collisional cross-section of the order of $10^{-19} \mathrm{~m}^{2}$ and $10^{-18} \mathrm{~m}^{2}$ for electrons and ions respectively and assuming a hydrogen gas at room temperature and a magnetic field of $B=0.1 \mathrm{~T}$, a inverted 
sheath is expected for pressure in the range 3-16 Pa. Our results can be useful for the interpretation of the Langmuir probe experiments in magnetized plasmas according to the collisionality ${ }^{42}$, as well as for the evaluation of the sheath width needed in sheath capacity calculations.

\section{ACKNOWLEDGMENTS}

This work was supported by the French National Research Agency (Agence Nationale de la Recherche) under project SHEAR ANR-19-CE30-033-01. It was also carried out within the framework of the Eurofusion consortium and of the French Federation for Magnetic Fusion Studies (FR-FCM). It received funding from the Euratom research and training programme 2019-2020 under Grant Agreement No. 633053. The views and opinions expressed herein do not necessarily reflect those of the European Commission.

\section{DATA AVAILABILITY}

The data that support the findings of this study are available from the corresponding author upon reasonable request.

\section{REFERENCES}

${ }^{1}$ C. D. Child, Phys. Rev. (Series I) 32, 492 (1911)

${ }^{2}$ I. Langmuir, Phys. Rev. 2, 450 (1913)

${ }^{3}$ Z. Sternovsky, S. Robertson, and M. Lampe, J. Appl. Phys. 94, 1374 (2003)

${ }^{4}$ T. E. Sheridan and J. Goree, Physics of Fluids B: Plasma Physics 3, 2796 (1991)

${ }^{5}$ M. S. Benilov, Plasma Sources Sci. Technol. 18, 014005 (2009)

${ }^{6} \mathrm{P}$. Chabert and N. Braithwaite, Physics of radio-frequency plasmas (Cambridge University Press, 2011) pp. 67-72

${ }^{7}$ D. Bohm, The Characteristics of Electrical Discharges in Magnetic Fields (A. Guthrie and

R. K. Wakerling, McGraw-Hill, New York, 1949) p. 77

${ }^{8}$ K. U. Riemann, J. Phys. D: Appl. Phys. 24, 493 (1991)

${ }^{9}$ D. D. Tskhakaya, B. Eliasson, P. K. Shukla, and S. Kuhn, Phys. Plasma 11, 3945 (2004)

${ }^{10}$ L. Tonks and I. Langmuir, Phys. Rev. 34, 876 (1929) 
${ }^{11}$ E. R. Harrison and W. B. Thompson, Proc. Phys. Soc. 74, 145 (1959)

${ }^{12}$ A. Caruso and A. Cavaliere, Il Nuovo Cimento 26, 1389 (1962)

${ }^{13}$ W. Schottky, Phys. Z. 25, 635 (1929)

${ }^{14}$ R. N. Franklin and J. Snell, Phys. Plasmas 8, 643 (2001)

${ }^{15}$ R. Chodura, Phys. Fluids 25, 1628 (1982)

${ }^{16}$ P. Stangeby, Phys. Plasmas 2, 702 (1995)

${ }^{17}$ K. U. Riemann, Phys. Plasma 1, 552 (1994)

${ }^{18}$ E. Ahedo, Phys. Plasma 4, 4419 (1997)

${ }^{19}$ P. Stangeby, Nuclear Fusion 52, 083012 (2012)

${ }^{20}$ D. Tskhakaya and S. Kuhn, J. Nuc. Mater. 313, 1119 (2003)

${ }^{21}$ J. Moritz, M. Lesur, E. Faudot, S. Devaux, S. Heuraux, and J. Ledig, Phys. Plasmas 26, 013507 (2019)

${ }^{22}$ D. D. Tskhakaya, P. K. Shukla, B. Eliasson, and S. Kuhn, Phys. Plasma 12, 103503 (2005)

${ }^{23}$ J. Moritz, E. Faudot, S. Devaux, and S. Heuraux, Phys. Plasmas 23, 062509 (2016)

${ }^{24}$ N. S. Krasheninnikova, X. Tang, and V. S. Roytershteyn, Phys. Plasma 17, 057103 (2010)

${ }^{25}$ K. Theilhaber and C. K. Birdsall, Phys. Rev. Lett. 62, 772 (1989)

${ }^{26}$ U. Daybelge and B. Bein, Phys. Fluids 24, 1190 (1981)

${ }^{27}$ D. L. Holland, B. D. Fried, and G. J. Morales, Phys. Fluids B 5, 1723 (1993)

${ }^{28}$ J. Kovai, T. Gyergyek, and M. erek, Eur. Phys. J. D 54, 383 (2009)

${ }^{29}$ R. Khaziev and D. Curreli, Phys. Plasmas 22, 043503 (2015)

${ }^{30}$ S. Devaux and G. Manfredi, Phys. Plasma 13, 083504 (2006)

${ }^{31}$ A. V. K. M. D. Campanell and I. D. Kaganovich, Phys. Rev. Lett. 108, 235001 (2012)

${ }^{32}$ D. Tskhakaya and S. Kuhn, J. Nuc. Mater. 337, 405 (2005)

${ }^{33}$ M. L. E. Kawamura, V. Vahedi and C. Birdsall, Plasma Sources Sci. Technol. 8, R45 (1999)

${ }^{34}$ P. Chabert, Plasma Sources Sci. Technol. 23, 065042 (2014)

${ }^{35}$ J. Moritz, E. Faudot, S. Devaux, and S. Heuraux, Phys. Plasmas 25, 013534 (2018)

${ }^{36}$ R. Procassini, C. Birdsall, and E. Morse, Physics of Fluids B: Plasma Physics 2, 3191 (1990)

${ }^{37}$ J. R. Myra, D. A. d'Ippolito, and M. J. Gerver, Nuclear Fusion 30, 845 (1990)

${ }^{38}$ A. Bergmann, Phys. Plasmas 1, 3598 (1994)

${ }^{39}$ I. Langmuir, Phys. Rev. 33, 954 (1929) 
${ }^{40}$ M. Campanell, Phys. Rev. E 88, 033103 (2013)

${ }^{41}$ S. D. Baalrud, N. Hershkowitz, and B. Longmier, Phys. Plasmas 14, 042109 (2007)

${ }^{42}$ J. Gunn, Phys. Plasmas 4, 4435 (1997) 